\title{
Dynamical evolution of titanium, strontium, and yttrium spots on the surface of the HgMn star HD 11753 ${ }^{\star}$
}

\author{
M. Briquet ${ }^{1 \star \star}$, H. Korhonen ${ }^{2}$, J. F. González ${ }^{3}$, S. Hubrig ${ }^{4}$, and T. Hackman ${ }^{5}$ \\ 1 Instituut voor Sterrenkunde, Katholieke Universiteit Leuven, Celestijnenlaan 200 D, 3001 Leuven, Belgium \\ e-mail: maryline@ster . kuleuven.be \\ 2 European Southern Observatory, Karl-Schwarzschild-Str 2, 85748 Garching bei München, Germany \\ 3 Instituto de Ciencias Astronomicas, de la Tierra, y del Espacio (ICATE), 5400 San Juan, Argentina \\ 4 Astrophysikalisches Institut Potsdam, An der Sternwarte 16, 14482 Potsdam, Germany \\ 5 Observatory, PO Box 14, 00014 University of Helsinki, Finland
}

Received 30 November 2009 / Accepted 25 February 2010

\section{ABSTRACT}

\begin{abstract}
Aims. We gathered about 100 high-resolution spectra of three typical HgMn (mercury-manganese) stars, HD 11753, HD 53244, and HD 221507, to search for slowly pulsating B-like pulsations and surface inhomogeneous distribution of various chemical elements. Methods. Classical frequency analysis methods were used to detect line profile variability and to determine the variation period. Doppler imaging reconstruction was performed to obtain abundance maps of chemical elements on the stellar surface.

Results. For HD 11753, which is the star with the most pronounced variability, distinct spectral line profile changes were detected for Ti, Sr, Y, Zr, and Hg, whereas for HD 53244 and HD 221507 the most variable line profiles belong to the elements Hg and Y, respectively. We derived rotation periods for all three stars from the variations of radial velocities and equivalent widths of spectral lines belonging to inhomogeneously distributed elements: $P_{\text {rot }}(\mathrm{HD} 11753)=9.54 \mathrm{~d}, P_{\text {rot }}(\mathrm{HD} 53244)=6.16 \mathrm{~d}$, and $P_{\text {rot }}(\mathrm{HD} 221507)=$ $1.93 \mathrm{~d}$. For HD 11753 the Doppler imaging technique was applied to derive the distribution of the most variable elements Ti, Sr, and Y using two datasets separated by $\sim 65$ days. Results of Doppler imaging reconstruction revealed noticeable changes in the surface distributions of Ti II, Sr II, and Y II between the datasets, indicating the hitherto not well understood physical processes in stars with radiative envelopes that cause a rather fast dynamical chemical spot evolution.
\end{abstract}

Key words. stars: chemically peculiar - stars: individual: HD 11753 - stars: individual: HD 53244 - stars: individual: HD 221507 stars: variables: general

\section{Introduction}

The mercury-manganese (HgMn) stars constitute a well-defined sub-class of chemically peculiar (CP) stars of the B7-B9 spectral types with $T_{\text {eff }}$ between 10000 and $15000 \mathrm{~K}$. These stars exhibit marked abundance anomalies of several elements: e.g., overabundances of $\mathrm{Hg}, \mathrm{Mn}, \mathrm{Ga}, \mathrm{Y}, \mathrm{Cu}, \mathrm{Be}, \mathrm{P}, \mathrm{Bi}, \mathrm{Sr}, \mathrm{Zr}$, and deficiencies of $\mathrm{He}, \mathrm{Al}, \mathrm{Zn}, \mathrm{Ni}$, Co. More than two thirds of them belong to spectroscopic binaries (Hubrig \& Mathys 1995). They are slow rotators $\left(\langle v \sin i\rangle=29 \mathrm{~km} \mathrm{~s}^{-1}\right.$, Abt 1972). There is no evidence that they would have strong largescaled organized magnetic fields. Their elemental overabundances/underabundances are believed to be due to radiativelydriven diffusion and gravitational settling.

In the H-R diagram many HgMn stars are located in the instability strip of the so-called slowly pulsating B (SPB) stars (see De Cat 2003, for a review on the latter), and the sophisticated models predict that pulsations should also be driven in HgMn stars (Turcotte \& Richard 2005). Searches for variability in this group of stars have been made mostly photometrically in the past, but without any success. Very recently, Alecian et al. (2009) discovered low amplitude (less than $1.6 \mathrm{mmag}$ )

* Based on observations obtained with the CORALIE Echelle Spectrograph on the 1.2-m Euler Swiss telescope, situated at La Silla, Chile.

$\star \star$ Postdoctoral Fellow of the Fund for Scientific Research, Flanders. periodic variations (4.3 and 2.53 days respectively, with harmonics) in two candidate $\mathrm{HgMn}$ stars by means of the high quality light curves provided by the CoRoT satellite. These variations are compatible with theoretically predicted pulsation periods. However, as stated by the authors, only spectroscopic datasets could help to conclusively establish or withdraw this pulsation interpretation. Currently, there is thus no observational proof of pulsation in HgMn stars.

The aspect of inhomogeneous distribution of some chemical elements over the surface of HgMn stars was for the first time discussed by Hubrig \& Mathys (1995). From the survey of $\mathrm{HgMn}$ stars in close spectroscopic binaries (SBs) it was suggested that some chemical elements might be inhomogeneously distributed on the surface, with in particular a preferential concentration of $\mathrm{Hg}$ along the equator. In close double-lined systems (SB2s), where the orbital plane has a small inclination to the line of sight, a rather large overabundance of $\mathrm{Hg}$ was found. By contrast, in stars with orbits almost perpendicular to the line of sight, mercury is not observed at all. The first indication of variability of the Hg II $3984 \AA$ and Y II $3983 \AA$ lines was reported for the HgMn SB2 system AR Aur by Takeda et al. (1979). Later, Wahlgren et al. (2001) and Adelman et al. (2002) showed that the $\mathrm{Hg}$ II $3984 \AA$ line of the primary component of $\alpha$ And varies with a 2.8 -d period. The spectral line variations were attributed to the surface inhomogeneous mercury distribution along the stellar equator, together with the stellar rotation period. 
Table 1. Atmospheric parameters (effective temperature, logarithm of the gravity, microturbulent velocity, and projected rotational velocity).

\begin{tabular}{ccccc}
\hline \hline & $T_{\text {eff }}(\mathrm{K})$ & $\log g$ & $\xi\left(\mathrm{km} \mathrm{s}^{-1}\right)$ & $v \sin i\left(\mathrm{~km} \mathrm{~s}^{-1}\right)$ \\
\hline HD 11753 & $10612 \pm 200$ & $3.79 \pm 0.10$ & $0.5 \pm 0.5$ & $14 \pm 0.5$ \\
HD 53244 & $13600 \pm 200$ & $3.40 \pm 0.03$ & $2.0 \pm 0.5$ & $35 \pm 0.5$ \\
HD 221507 & $12476 \pm 200$ & $4.13 \pm 0.10$ & $0.0 \pm 0.5$ & $25 \pm 0.5$ \\
\hline
\end{tabular}

Notes. Taken from Dolk et al. (2003) for HD 11753 and HD 221507, and from Woolf \& Lambert (1999) for HD 53244.

Recently, Kochukhov et al. (2005) found clear signatures of surface mercury spots in two rapidly rotating HgMn stars by analysing the Hg II $3984 \AA$ line profiles. Variability of spectral lines associated to larger number of chemical elements were discovered for the first time by Hubrig et al. (2006a) for the primary component of the eclipsing binary AR Aur. The strongest variations were found for the chemical elements $\mathrm{Pt}, \mathrm{Hg}, \mathrm{Sr}, \mathrm{Y}$, $\mathrm{Zr}, \mathrm{He}$, and $\mathrm{Nd}$. The first Doppler maps for the elements $\mathrm{Mn}$, $\mathrm{Sr}, \mathrm{Y}$, and $\mathrm{Hg}$ were recently presented by Savanov et al. (2009). The study of Hubrig et al. (2008) suggests that spectral variability of various chemical elements is indeed observed in most HgMn stars.

We present the first observational study based on a substantial number of spectra, more than one hundred, obtained with the CORALIE échelle spectrograph attached to the $1.2 \mathrm{~m}$ Leonard Euler telescope in La Silla in Chile. The selected targets, the single-lined (SB1) spectroscopic binaries HD 11753 ( $\phi$ Phe, $V=$ $5.1 \mathrm{mag}, \mathrm{B} 9 \mathrm{p})$ and HD 53244 ( $\gamma \mathrm{CMa}, V=4.1 \mathrm{mag}, \mathrm{B} 8 \mathrm{II})$, and the star HD 221507 ( $\beta \mathrm{Scl}, V=4.4 \mathrm{mag}, \mathrm{B} 9.5 \mathrm{IVmnpe})$ were chosen as the brightest known southern $\mathrm{HgMn}$ stars visible during the periods of observation. The goal of the presented spectroscopic study was twofold: to search for stellar pulsations and/or surface inhomogeneous distribution of various chemical elements. Our observations and data reduction are presented in Appendix A.

\section{Line profile variability}

Atmospheric parameters and abundances of several elements of selected HgMn stars were studied in the past by Smith \& Dworetsky (1993), Woolf \& Lambert (1999) and Dolk et al. (2003). The stellar parameters for our three studied stars are given in Table 1 . Their position in a $\left(\log T_{\text {eff }}, \log g\right)$-diagram is presented in Fig. 1. The star HD 221507 is located in the SPB instability strip, whereas HD 11753 and HD 53244 are located outside this strip. According to theoretical models (e.g. Miglio et al. 2007) HD 221507 is expected to exhibit SPB-like pulsations. To search for variability we studied the behaviour of the radial velocities and equivalent widths of the spectral lines of various elements. We used the Lomb-Scargle method (Scargle 1982) and the phase dispersion minimization method (Stellingwerf 1978). Additionally, we performed a two-dimensional frequency analysis, which is available in FAMIAS (Frequency Analysis and Mode Identification for Asteroseismology) (Zima 2008).

Among the studied stars, the spectra of HD 11753 exhibit the most prominent variability. This was most clearly detectable for the elements $\mathrm{Ti}, \mathrm{Sr}$, and $\mathrm{Y}$. The Fe lines, by contrast, show no profile variations. For that reason, we determined the stellar radial velocity (RV) by measuring $16 \mathrm{Fe}$ lines free of blends. The star HD 11753 is known as a single-lined binary star, but its orbital parameters are not well known. According to our observations the orbital period would be long. In fact,

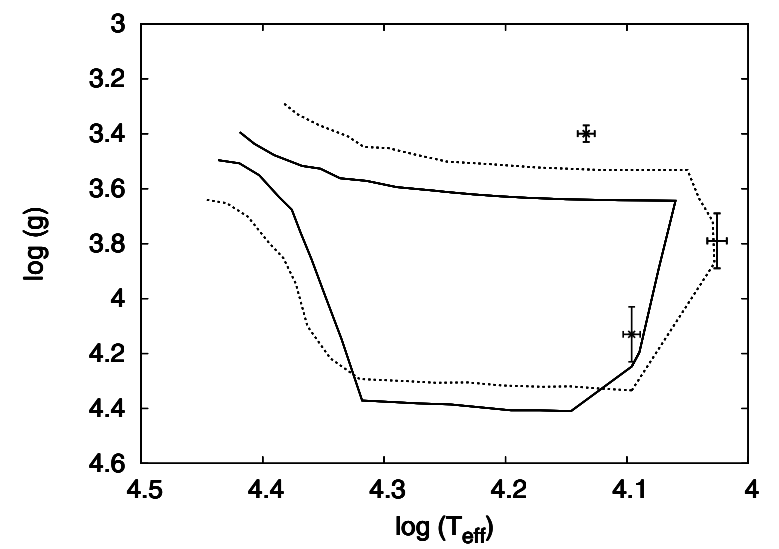

Fig. 1. Position of the studied stars in a $\left(\log T_{\text {eff }}, \log g\right)$-diagram. From left to right, the error boxes represent HD 53244, HD 221507, and HD 11753 . The full and dashed lines represent the boundary of the theoretical SPB instability strip for a metallicity $Z=0.02$ and $Z=0.01$, respectively (taken from Miglio et al. 2007).

all our RV measurements, which extend over 10 months, range from 14.1 to $14.5 \mathrm{~km} \mathrm{~s}^{-1}$. In particular for the two first datasets (76 and 28 observations, respectively) the mean RVs are $14.24 \pm 0.01 \mathrm{~km} \mathrm{~s}^{-1}(\mathrm{rms}$ (root mean square $\left.)=0.05 \mathrm{~km} \mathrm{~s}^{-1}\right)$ and $14.36 \pm 0.01 \mathrm{~km} \mathrm{~s}^{-1}\left(\mathrm{rms}=0.05 \mathrm{~km} \mathrm{~s}^{-1}\right)$. Considering the small dispersion and the absence of any trend with the rotational velocity found from Y lines, we adopted these values as the stellar RV for the observations of the corresponding datasets. For HD 53244 our RVs present an rms of $0.2 \mathrm{~km} \mathrm{~s}^{-1}$ without significant differences between different runs. Therefore we did not apply an RV correction other than the subtraction of the mean RV.

For HD 11753, the radial velocities and equivalent widths of the Ti, Sr, and Y lines were found to vary with the period $P=$ $9.54 \mathrm{~d}$. In the spectra of HD 53244 the variations are apparent for the elements $\mathrm{Hg}$ and $\mathrm{Mn}$ with the $P=6.16 \mathrm{~d}$, while in the spectra of HD 221507 variations associated to $\mathrm{Hg}, \mathrm{Mn}$, and Y are detected, indicating the period $P=1.93 \mathrm{~d}$.

For all three observed stars the behaviour of the line profiles is different for different elements. Moreover, spectral lines associated to certain elements, like silicon, are constant. These characteristics do not support the SPB-like pulsation interpretation (De Cat 2001). We thus conclude that the determined periods correspond to rotation periods of studied HgMn stars and the detected line profile variability is caused by inhomogeneous distribution of chemical elements on their stellar surface. Apart from two other HgMn stars, $\alpha$ And and AR Aur, no other rotation period determinations for $\mathrm{HgMn}$ stars were published before this study. In Figs. 2 and 3 we display time-series spectra phased on the stellar rotation periods around selected lines of various elements in all three stars. The images were created by averaging all the spectra within the phase interval $\phi \pm 0.04$ for each phase $\phi$.

\section{Surface chemical inhomogeneities by Doppler imaging reconstruction}

For the star HD 11753, which exhibits the most distinct spectral line profile variations for several elements, we used the Doppler imaging technique to reconstruct the surface distributions of $\mathrm{Ti}$, $\mathrm{Sr}$, and Y. The elements Ti and Y have numerous transitions in the observed optical spectral region allowing us to select unblended spectral lines which show strong variability.

The two sets of observations of HD 11753 obtained in 2000 Sep. 28-Oct. 11 (set1) and in 2000 Dec. 02-Dec. 15 

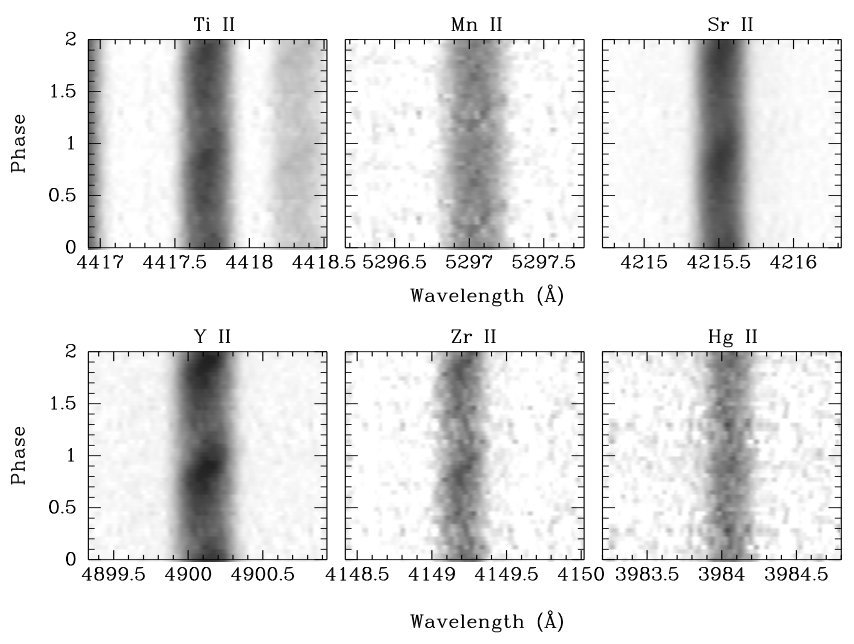

Fig. 2. Spots on the surface of HD 11753: time-series spectra phased on the stellar rotation period of 9.54 days.

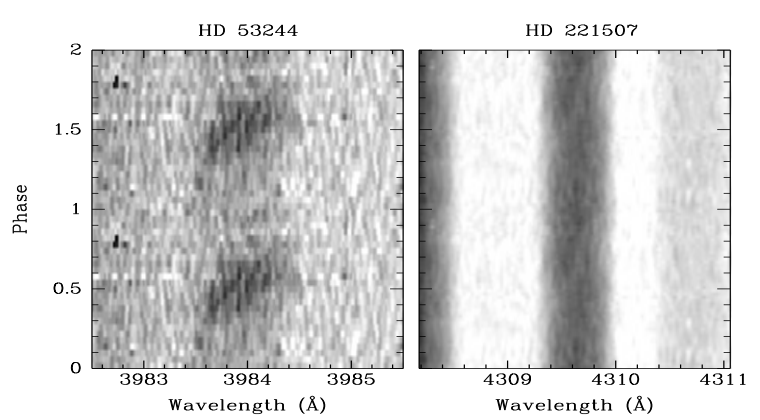

Fig. 3. Left: inhomogeneous distribution of $\mathrm{Hg}$ II on the surface of HD 53244 apparent in the time-series spectra around Hg II $\lambda 3984$ phased on the rotation period of 6.16 days. Right: inhomogeneous distribution of Y II on the surface of HD 221507 apparent in time-series spectra around the spectral line of Y II $\lambda 4310$ phased on the rotation period of 1.93 days.

(set2) consist of 76 and 28 observations, respectively, evenly spread over the stellar rotation cycle. Using two separate surface abundance maps based on observations of two data sets that are on average 65 days apart allowed us to obtain important information on the temporal evolution of elemental surface inhomogeneities. The Doppler imaging technique takes advantage of the partial resolution of the stellar surface provided by the rotational Doppler effect, inverting a line profile time series into a 2D map of the stellar surface. The inversion of time series of spectroscopic observations is based on regularised imagereconstruction procedures implemented in the Doppler imaging code INVERS7PD written by Piskunov (see, e.g., Piskunov et al. 1990) and modified by Hackman (2001).

The observations were compared to a grid of local line profiles calculated with the SPECTRUM spectral synthesis code (Gray \& Corbally 1994) and Kurucz model atmospheres (Kurucz 1993). The local line profiles were calculated for 10 limb angles, and the stellar parameters were fixed to the values given in Table 1. For inclination, $v \sin i$, and microturbulence inversion using several different values was carried out to determine the value that best fitted the observations. As best values for Ti and Y surface distribution reconstruction we obtained $v \sin i=13.5 \mathrm{~km} \mathrm{~s}^{-1}, i=53^{\circ}$, and $\xi=0.5 \mathrm{~km} \mathrm{~s}^{-1}$. On the other hand, the best fit for the reconstruction of the Sr distribution was achieved for $v \sin i=12.3 \mathrm{~km} \mathrm{~s}^{-1}$. The discrepancies in the $v \sin i$ values are very likely related to the Sr vertical abundance
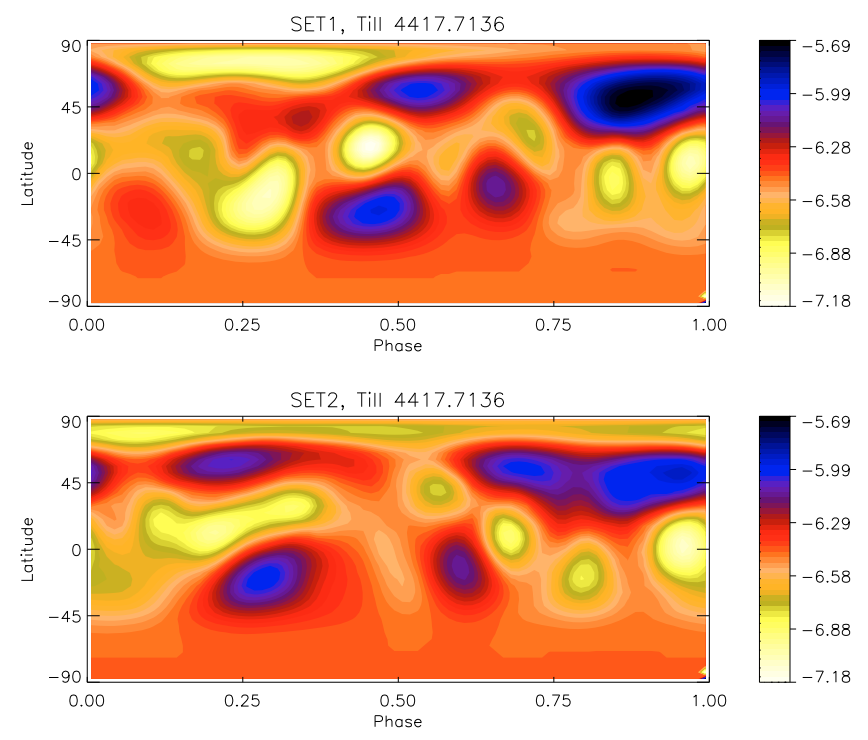

Fig. 4. Ti abundance map of HD 11753 obtained from Ti II line $4417.7136 \AA$ for set 1 and set 2 . The colour indicates the abundance with respect to the total number density of atoms and ions.

stratification, which is frequently observed in chemically peculiar stars (e.g. Kochukhov et al. 2006). For each studied element a grid of abundances spanning from -4.0 to -9.5 was calculated, using a step of 0.5 in abundance. These abundances are in the scale used by SPECTRUM and are thus expressed with respect to the total number density of atoms and ions, and not with respect to hydrogen, with the log of the abundance of hydrogen set equal to 12.0 .

The Ti II line chosen for the inversions is $4417.7136 \AA$, which has an excitation potential of $1.237 \mathrm{eV}$. Chemical maps were separately recovered for both datasets (set1 and set2). As can be seen in Fig. 4, the Ti abundance does not exhibit a distinct ring structure around the stellar equator as was found for the $\mathrm{Hg}$ distribution in the $\mathrm{HgMn}$ star $\alpha$ And (Adelman et al. 2002). The maps in general show a surface abundance that is higher than the solar abundance of $\mathrm{Ti},-7.02$, and have an average abundance of -6.47 . Two main structures are well noticeable on the surface: a high abundance spot at high latitudes $\left(40^{\circ}-75^{\circ}\right)$ spanning the phases $0.75-1.00$, with an extension towards and beyond the phase 0.5 forming a half ring, and a lower abundance spot in the polar regions at the phases $0.1-0.5$. Also, some discrete spots of lower and higher abundance appear in the equatorial region. The Ti lower abundance spot at phases $0.1-0.5$ is less prominent in set 2 than in set1. Also the spot configuration of the high abundance high latitude half ring of $\mathrm{Ti}$ at the latitudes $40^{\circ}-75^{\circ}$ changes between the two sets.

The maps obtained from the Y II line $4900.1200 \AA$ with an excitation potential of $1.033 \mathrm{eV}$ is presented in Fig. 5. The maps show a high abundance region at phases $0.5-1.0$ extending from the latitude $45^{\circ}$ to the pole, with an extension to the equator around phase 0.8 . The $\mathrm{Y}$ abundance distribution shows a high latitude lower abundance spot around phases $0.2-0.4$, similarly to the Ti abundance maps. Some lower and higher abundance spots are also seen at the equatorial region. The average abundance of the $\mathrm{Y}$ maps is -7.01 , which is significantly higher than the solar abundance of -9.80 . We note that all the features revealed in the maps show abundances that are higher than the solar abundance of Y. Similar to the Ti maps, we observed in the $\mathrm{Y}$ maps that the lower abundance high latitude feature at phases $0.2-0.4$ becomes more extended and less prominent 

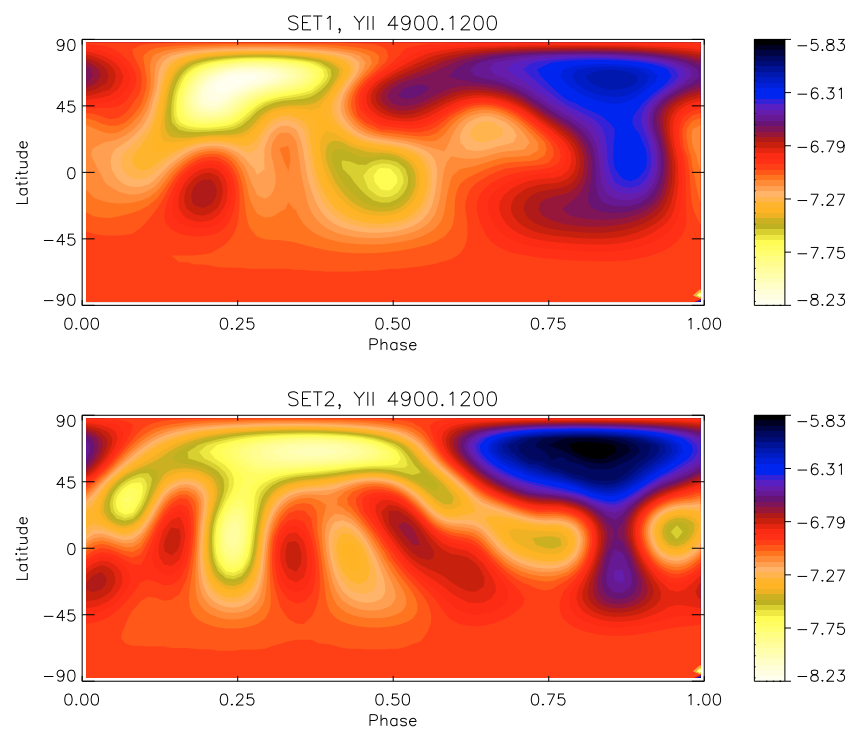

Fig. 5. As in Fig. 4, but now using the Y II line $4900.1200 \AA$.
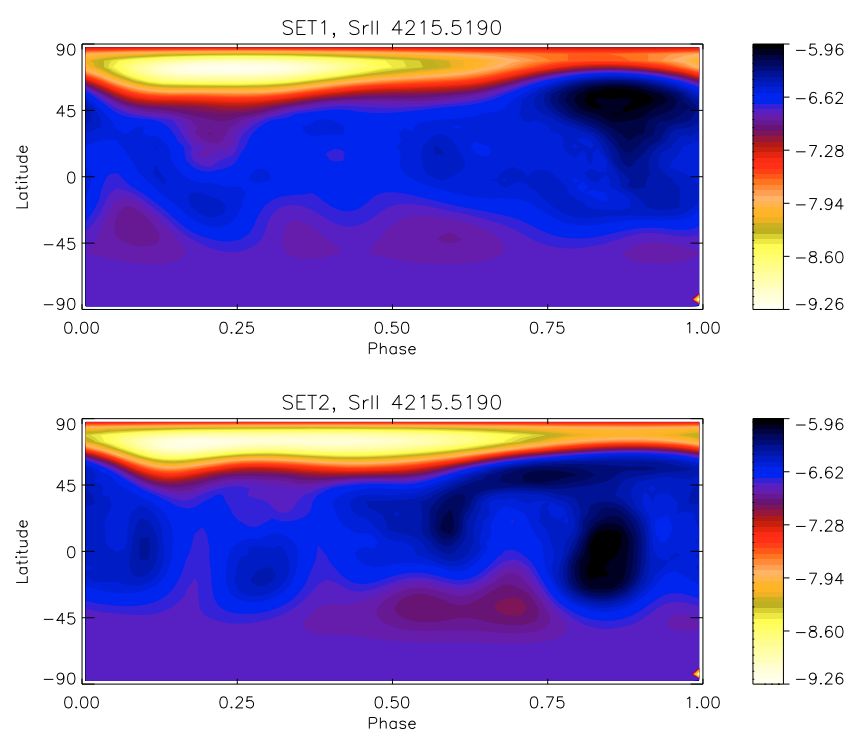

Fig. 6. As in Fig. 4, but now using the Sr II line $4215.5190 \AA$ A.

in set2. Additionally, the abundance of the high abundance spot at phases $0.6-1.0$ increases.

The $\mathrm{Sr}$ maps based on the modelling of the $\mathrm{Sr}$ II $4215.5190 \AA$ line reveal similarities in the distribution of overabundant and underabundant features, though the similarity is more pronounced in the Sr and Y maps. In Fig. 6 the equatorial and polar belt-like $\mathrm{Sr}$ structures resemble rather well those of Y. The time evolution of the chemical spots is also seen in the $\mathrm{Sr}$ maps, with the lower abundance high latitude feature at phases $0.1-0.5$ becoming more extended in set2, and the high abundance feature of the phases $0.8-1.0$ moving towards the equator and phases 0.5 . The average abundance of the $\mathrm{Sr}$ maps is -6.89 , which exceeds the solar abundance of -9.07 .

One has to keep in mind though that set2 has a gap at the phases $0.187-0.391$. This phase gap can affect the achieved resolution on the surface at these phases, and can especially hamper the exact determination of the latitudes. Still, the changes between the two sets are seen from the maps at other phases, and also from the line profiles themselves as shown in Appendix C.

\section{Discussion}

All Ti, Sr, and Y abundance maps reveal a structure reminiscent of broken rings of low and high abundance. This elemental distribution is to some extent similar to the maps previously reconstructed for another HgMn star, AR Aur (Savanov et al. 2009), where the elements $\mathrm{Mn}, \mathrm{Y}, \mathrm{Sr}$, and $\mathrm{Hg}$ show abundance concentration in equatorial and polar features. Typically, inhomogeneous chemical abundance distributions are observed only on the surface of magnetic chemically peculiar stars with large-scale organised magnetic fields. In these stars, the abundance distribution of certain elements is non-uniform and non-symmetric with respect to the rotation axis. A magnetic field of the order of a few hundred Gauss was detected in hydrogen lines of four HgMn stars by Hubrig et al. (2006b) using low-resolution $(R=2000)$ circular polarisation spectra obtained with FORS 1 at the VLT. This small sample of HgMn stars also included the spectrum variable $\mathrm{HgMn}$ star $\alpha$ And with a magnetic field of the order of a few hundred Gauss. On the other hand, highresolution spectropolarimetric spectra of some $\mathrm{HgMn}$ stars, including $\alpha$ And, were used in studies of Shorlin et al. (2002) and Wade et al. (2006), where no detection was achieved using all metal lines together in the least-squares deconvolution multiline profile. Although strong large-scale magnetic fields have not generally been found in HgMn stars, it has never been ruled out that these stars might have tangled magnetic fields of the order of a few thousand Gauss with no net longitudinal component (e.g., Mathys \& Hubrig 1995; Hubrig et al. 1999; Hubrig \& Castelli 2001). It is of interest that magnetohydrodynamical simulations by Arlt et al. (2003), which combine a poloidal magnetic field and differential rotation can produce a magnetic field topology that is similar to the broken elemental ring structures seen in HD 11753 and AR Aur. These simulations and their implication have been recently discussed by Hubrig et al. (2008).

The abundance maps of HD 11753 presented in this work exhibit clear differences between the surface abundance distribution of Ti, Sr, and Y. We also detected clear differences in the spot configurations obtained from the same lines but for different data sets, which indicates a rather fast dynamical evolution of the abundance distribution with time. Kochukhov et al. (2007) discovered mercury clouds in the atmosphere of a HgMn star $\alpha$ And that showed secular changes with a time period of 2-4 years. In our analysis, using two datasets separated by $\sim 65$ days, we reveal that the changes in the chemical spot configuration of HD 11753 appear much faster and can already be detected at a time scale of months.

The results reported in this paper open up new perspectives for our knowledge and understanding of $\mathrm{HgMn}$ stars. Different dynamical processes take place in stellar radiation zones. An interaction between the differential rotation, the magnetic field, and the meridional circulation could possibly play a role in the generation of dynamical evolution of chemical spots. From the comparison of maps we find that it is possible that the $\mathrm{Y}$ and Sr distributions show indications of an increasing rotation rate towards the rotation pole, so-called differential rotation of antisolar type. On the other hand, further analyses of the elemental surface distribution in a larger sample of $\mathrm{HgMn}$ stars should be carried out before the implication of these new results can be discussed in more detail.

Acknowledgements. We thank our colleagues from the Institute of Astronomy of Leuven University, who contributed to the gathering of these data. 

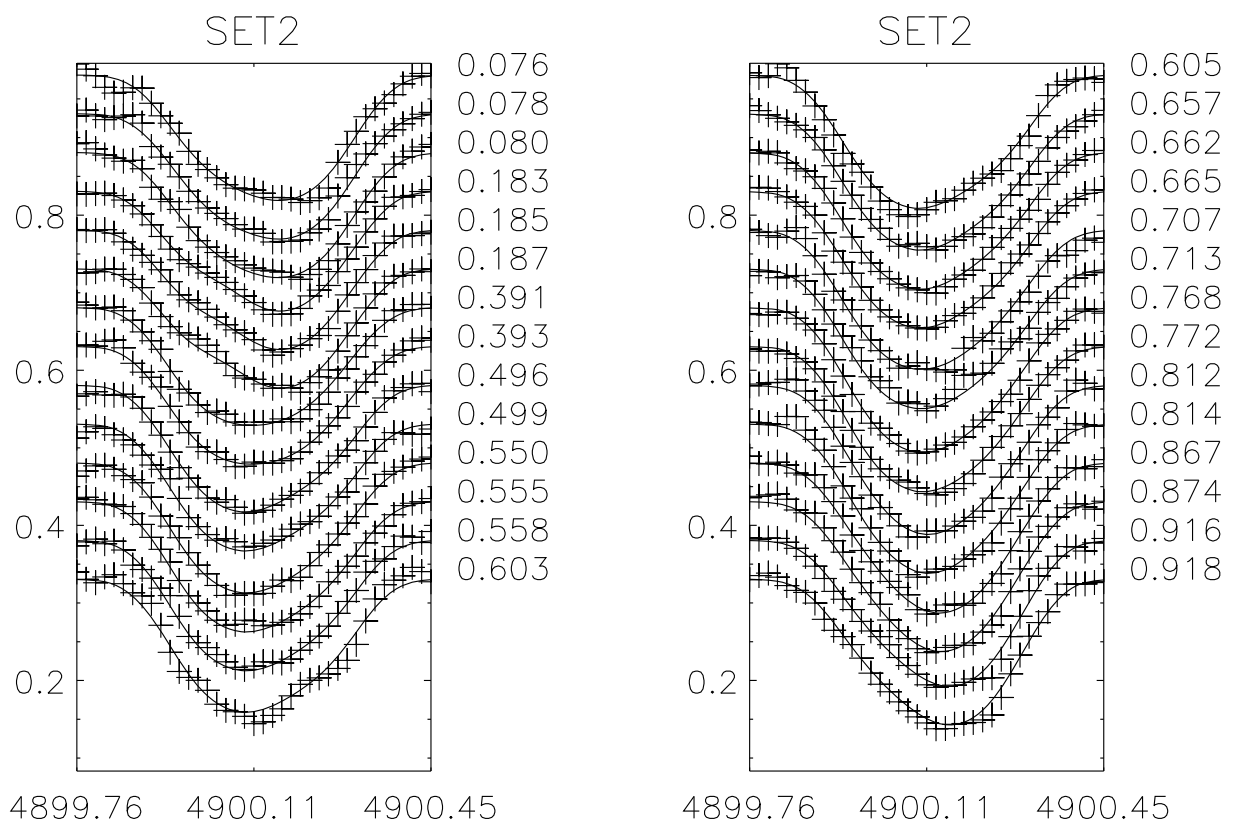

Fig. B.1. Set2 Y II $4900.1200 \AA$ A spectra of HD 11753 used in the Doppler imaging. Calculated and observed spectral lines are shown by lines and crosses, respectively. The numbers on the right side of the plots give the phase for each spectrum.

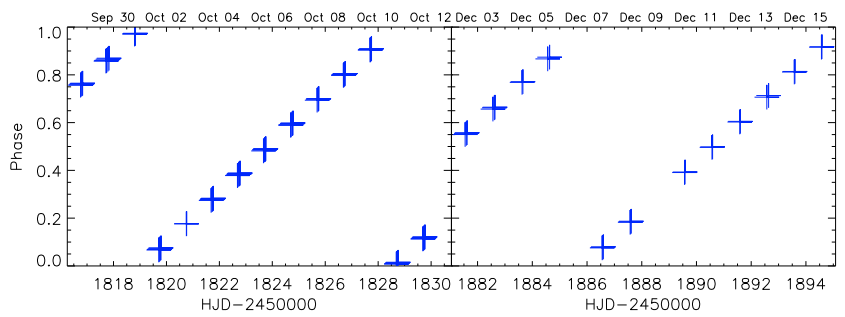

Fig. A.1. Phases of the HD 11753 observations for both sets (set1 on the left).

\section{Appendix A: Observations and data reduction}

Our spectroscopic data were obtained with the CORALIE échelle spectrograph attached to the $1.2 \mathrm{~m}$ Leonard Euler telescope in La Silla in Chile. In total, we gathered 113, 103, and 105 useful spectra for HD 11753, HD 53244, and HD 221507, respectively. The observing logbook is given in Table A.1 and the obtained phases for HD 11753 in Fig. A.1. The wavelength domain of the CORALIE fibre-fed spectrograph ranges from 3875 to $6820 \AA$ recorded on 68 orders. The CCD camera is a $2 \mathrm{k} \times 2 \mathrm{k}$ CCD with pixels of $15 \mu \mathrm{m}$. CORALIE reaches a spectral resolution of 50000 with a 3 pixel sampling.

To search for variability due to pulsation in line profiles we used rather short integration times to obtain a $\mathrm{S} / \mathrm{N}$ ratio of about 100 near the wavelength $4130 \AA$, which is the position of a prominently present $\mathrm{Si}$ II doublet. This spectral region is well-suited for studying line profile variability of SPB stars (e.g., De Ridder et al. 2002).

We used the online reduction package available for the CORALIE spectrograph based on the method of Baranne et al. (1996). The process involves the usual steps of de-biasing, flatfielding, background subtraction and wavelength calibration by means of measurements of a ThAr calibration lamp. We did a more precise correction for the pixel-to-pixel sensitivity variations by using all available flat fields obtained during the night instead of using only one flat field, as is done by the on-line
Table A.1. Logbook of our spectroscopic observations of the three HgMn stars.

\begin{tabular}{cccc}
\hline \hline & $N$ & HJD begin & HJD end \\
\hline HD 11753 & 76 & 51816 & 51829 \\
& 28 & 51881 & 51894 \\
& 6 & 51942 & 51948 \\
& 3 & 52111 & 52123 \\
\hline HD 53244 & 56 & 51816 & 51829 \\
& 18 & 51881 & 51893 \\
& 29 & 51940 & 51953 \\
\hline HD 221507 & 68 & 51816 & 51829 \\
& 31 & 51881 & 51894 \\
& 6 & 52110 & 52122 \\
\hline
\end{tabular}

Notes. $N$ denotes the number of observations and the HJD is given with respect to $\mathrm{HJD}_{0}=2400000$.

reduction procedure. After a correction to the heliocentric frame, the spectra were normalised using a cubic spline fit.

\section{Appendix B: Details on Doppler imaging}

The average S/N ratio of the HD 11753 observations for set 1 and set2, measured at $4400 \AA$ are 132 and 143, respectively. This means that in Doppler imaging a smaller deviation than this should not be achieved between the model and the observations. In the Tikhonov regularisation used by INVERS7PD the regularisation has to be chosen in a way that the noise in the observations is not mapped. For HD 11753 the deviation between the model and observations for the set 1 is between $0.750 \%$ (Y II $4900.120 \AA$ ) and $0.893 \%$ (Sr II $4215.5190 \AA$ ), and for the set2 between $0.628 \%$ (Y II $4900.1200 \AA$ ) and $0.803 \%$ (Sr II $4215.5190 \AA$ ). These values are comparable to the average noise level in the observations. One has to also keep in mind that the noise level in the blue part is higher than in the red part of the spectrum. Furthermore, the comparison of the model and observations shows that the line profiles are well fitted without 

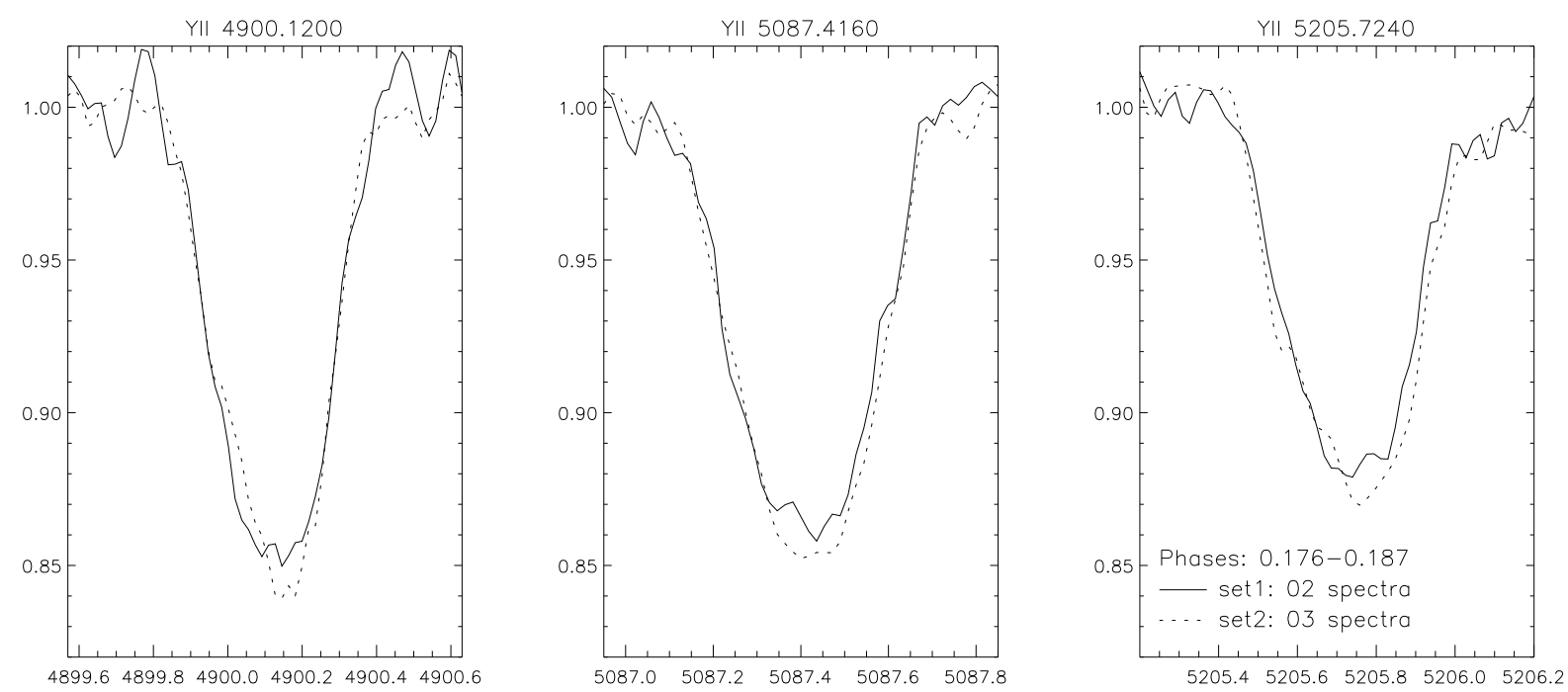

Fig. C.1. Mean Y II line profiles from close-by phases for set1 (phases 0.176-0.177) and set2 (phases 0.183-0.187). The two mean spectra are plotted for three YII lines, which have similar excitation energies: $4900.1200 \AA$, $5087.4160 \AA$ and YII $5205.7240 \AA$. In the plots set1 is given by the solid line and set 2 by the dashed line.

the model following the noise. An example of the line profile fits is shown in Fig. B.1 for the Y II $4900.1200 \AA$ A line of set2.

\section{Appendix C: Line variability}

The changes between the two sets are also seen when looking at the line profiles at the phases where the largest differences in the maps occur. Figure C. 1 shows averaged spectra of very closeby phases from set1 (mean of two spectra, phases: 0.176-0.177) and set2 (mean of three spectra, phases: 0.183-0.187). The mean profiles for the two sets are shown for three Y II lines of similar strength and excitation energy (4900.1200 ̊, 5087.4160 ̊ and $5205.7240 \AA$ ). As can be seen all three lines show changes between the two sets that are similar and in-line with the changes seen in the Doppler images, i.e., the Y II abundance is higher for set 2 than set 1 at these phases.

\section{References}

Abt, H. A., Chaffee, F. H., \& Suffolk, G. 1972, ApJ, 175, 779 Adelman, S. J., Gulliver, A. F., Kochukhov, O. P., \& Ryabchikova, T. A. 2002, ApJ, 575, 449

Alecian, G., Gebran, M., Auvergne, M., et al. 2009, A\&A, 506, 69

Arlt, R., Hollerbach, R., \& Rüdiger, G. 2003, A\&A, 401, 1087

Baranne, A., Queloz, D., Mayor, M., et al. 1996, A\&A, 119, 373

De Cat, P. 2001, Ph.D. Thesis, KULeuven, Belgium

De Cat, P. 2003, Ap\&SS, 284, 37
De Cat, P., Briquet, M., Aerts, C., et al. 2007, A\&A, 463, 243

De Ridder, J., Dupret, M.-A., Neuforge, C., \& Aerts, C. 2002, A\&A, 385, 572

Dolk, L., Wahlgren, G. M., \& Hubrig, S. 2003, A\&A, 402, 299

Gray, R. O., \& Corbally, C. J. 1994, AJ, 107, 742

Hackman, T., Jetsu, L., \& Tuominen, I. 2001, A\&A, 374, 171

Hubrig, S., \& Mathys, G. 1995, Com. Ap, 18, 167

Hubrig, S., \& Castelli, F. 2001, A\&A, 375, 963

Hubrig, S., Castelli, F., \& Wahlgren, G. M. 1999, A\&A, 346, 139

Hubrig, S., González, J. F., Savanov, I., et al. 2006a, MNRAS, 371, 1953

Hubrig, S., North, P., Schöller, M., \& Mathys, G. 2006b, AN, 327, 289

Hubrig, S., González, J. F., \& Arlt, R. 2008, CoSka, 38, 415

Kochukhov, O., Piskunov, N., Sachkov, M., \& Kudryavtsev, D. 2005, A\&A, 439, 1093

Kochukhov, O., Tsymbal, V., Ryabchikova, T., Makaganyk, V., \& Bagnulo, S. 2006, A\&A, 460, 831

Kochukhov, O., Adelman, S. J., Gulliver, A. F., \& Piskunov, N. 2007, Nature Phys., 3, 526

Kurucz, R. L. 1993, Kurucz CD, 13

Mathys, G., \& HUbrig, S. 1995, A\&A, 293, 810

Miglio, A., Montalbán, J., \& Dupret, M.-A. 2007, MNRAS, 375, 21

Piskunov, N. E., Tuominen, I., \& Vilhu, O. 1990, A\&A, 230, 363

Savanov, I. S., Hubrig, S., González, J. F., \& Schöller, M. 2009, IAU Symp., 259, 401

Scargle, J. D. 1982, ApJ, 263, 835

Smith, K. C., \& Dworetsky, M. M. 1993, A\&A, 274, 335

Shorlin, S. L. S., Wade, G. A., Donati, J.-F., et al. 2002, A\&A, 392, 637

Stellingwerf, R. F. 1978, ApJ, 224, 953

Takeda, Y., Takada, M., \& Kitamura, M. 1979, PASJ, 31, 821

Turcotte, S., \& Richard, O. 2005, EAS Publ. Ser., 17, 357

Wade, G. A., Auriere, M., Bagnulo, S., et al. 2006, A\&A, 451, 293

Wahlgren, G. M., Ilyin, I., \& Kochukhov, O. 2001, AAS, 33, 1506

Woolf, V. M., \& Lambert, D. L. 1999, ApJ, 521, 414

Zima, W. 2008, CoAst, 157, 387 